\title{
Gerascofobia - o medo de envelhecer na contemporaneidade
}

\author{
Bárbara Slonski Delboni*, Stephanie Bittencourt Joaquim*, \\ Katia Simone Ploner", Luiz Arthur Rangel Cyrino"**
}

\section{Resumo}

O estudo tem o intuito de contribuir com discussões relacionadas ao advento da terceira idade e suas implicações em relação à ideologia narcisista social em que vivemos hoje, induzida pela mídia, salientando, ainda, a importância desse tema para outros campos de estudo, como a psicologia, a gerontologia e a geriatria. A pesquisa realizada é uma revisão bibliográfica referente à ascensão da população idosa na pirâmide sociodemográfica brasileira e a sua experiência do processo de envelhecimento, e no conceito em que é vivido de maneira estigmatizada, passa a representar uma ameaça à aceitação de si, tornando os indivíduos vulneráveis a sofrimentos psíquicos de toda ordem e até mesmo a patologias. Para um gerascofóbico o despertar da doença é pertinente a um temor do que lhes espera na maturidade, com isso, cria-se um medo do decorrer do ciclo da vida, não compreendendo de forma natural as consequências que o processo de envelhecer aborda, arriscando de diversas maneiras retardar os sinais da velhice.
Percebe-se, então, a presença de sintomas fisiológicos reais de ansiedade patológica, que se caracteriza de um medo exacerbado, podendo afetar a vida de um indivíduo, causando tensões, angústias e preocupações exageradas, levando à busca, a qualquer custo, da "fonte da juventude", sem considerar os riscos relacionados a essa prática.

Palavras-chave: Gerascofobia. Fobia. Envelhecimento. Transtorno de ansiedade.

\section{Introdução}

A passagem por diferentes fases da vida é um processo pelo qual todos, inevitavelmente, passam. A ciência desse processo, contudo, não evita que os medos inerentes a ele instalem-se nos indivíduos. Estamos cada vez mais preocupados com o envelhecimento e os seus danos.

* Acadêmicas do curso de Psicologia da Universidade da Região de Joinville, Univille. E-mails: barbarasboni@ hotmail.com; teffy.bittencourt@hotmail.com.

** Mestre em psicologia social e da personalidade. Professora do curso de Psicologia da Universidade do Vale do Itajaí, Univali. Endereço para correspondência: Rua Princesa Isabel, 238, sala 414, Centro, CEP:89201904, Joinville, (SC). Email: rangel7@uol.com.br.

**** Mestre em Neurociências. Professor de psicofisiologia e psicofarmacologia do departamento de Psicologia da Universidade da Região de Joinville, Univille.

$\rightarrow$ http://dx.doi.org/10.5335/rbceh.2013.3320 
No entanto, existem indivíduos que se destacam pela angústia e ansiedade com que nutrem essas alterações. Souza et al. (2007) mencionam que o envelhecimento e, em particular, a velhice podem ser considerados momentos de crise no ciclo vital, uma vez que representam situações de mudanças (bio-psico-sociais), requerendo adaptações a essa etapa da vida, tanto do ser que envelhece, como dos sujeitos com quem convive.

A presente discussão justifica-se pelo aspecto sociodemográfico de países industrializados, tomando, por exemplo, o Brasil, no qual o envelhecimento da população cresce exponencialmente de acordo com a expectativa de vida. Para Veras (2009), o aumento da população senil é um acontecimento mundial. No caso do Brasil, a transição da faixa etária predominante na população ocorre de maneira rápida, visto que as projeções mais conservadoras apontam o Brasil, para 2020, como o sexto país do mundo em quantidade de idosos, com uma população superior a trinta milhões de pessoas idosas.

Acredita-se que, na era do capitalismo, a mídia tem propagado a visão de envelhecimento como sinônimo da decadência do ser humano, atrelando o consumo à garantia de jovialidade eterna e de status. Assim, a estética ganha campo quando é relacionada à garantia de felicidade. Borges et al. (2012) elucidam que os limites do corpo desistiram de ser poupados e esse é aceito, então, como produto. A ansiedade que o envelhecer gera, pode ser acentuada pela mídia e a preocupação com o entardecer da vida evolui para um transtorno de ansiedade mais específico, como uma fobia, chamada de gerascofobia.
Objetivamos com o estudo, contribuir para a ciência psicológica, em relação ao advento da terceira idade e suas implicações relacionadas à ideologia narcisista social induzida pela mídia, salientando, também, a importância desse tema para outros campos de pesquisa, como a gerontologia e a geriatria. Procuramos definir o termo gerascofobia como uma espécie de fobia, a qual se refere ao medo persistente anormal e injustificado de envelhecer, acarretando infelicidades, independente da boa saúde e da posição financeira do sujeito fóbico.

\section{Ansiedade: definição e características}

Os sentimentos ansiosos estão presentes na vida de todos os seres humanos e, segundo Nardi et al. (2012), os transtornos de ansiedade não são um problema do nosso tempo: vários textos literários e mitos da antiguidade demonstram que os sintomas do que chamamos, atualmente, de transtornos de ansiedade foram observados há muito tempo. Silva (2011) afirma que a ansiedade é uma reação natural do organismo, ou seja, um extinto de sobrevivência desenvolvido para que o ser humano reaja diante do perigo. Até certo ponto, é necessário para a sobrevivência. Porém, quando a ansiedade é desproporcional, torna-se patológica, desencadeando uma série de sofrimentos e problemas de ordem social.

De acordo Zamignani e Banaco (2005), o termo ansiedade refere-se a eventos bastante diversos, tanto no que diz respeito a estados internos do falante, quanto a processos comportamentais que produzem esses estados internos. Quem 
sofre de ansiedade passa por angústias, medos e receios, cujas origens são difíceis de identificar com clareza. Dalgalarrondo (2000) alega que são frequentes os sintomas como insônia, dificuldade em relaxar, angústia constante, irritabilidade aumentada e dificuldade em concentrar-se. Além de sintomas físicos como cefaleia, dores musculares, dores ou queimação no estômago, taquicardia, tontura, formigamento e sudorese fria.

Segundo dados da Organização Mundial de Saúde (OMS), sintomas de ansiedade patológica estão presentes em um número cada vez maior de pessoas nos dias atuais, principalmente nos grandes centros urbanos. Conforme Wittchen e Fehm (2001), os portadores de transtornos de ansiedade apresentam uma redução significativa da qualidade de vida, inclusive com maiores taxas de comorbidades, ou seja, a associação de duas ou mais patologias em um mesmo paciente. Bourne (2008) afirma que o ambulatório de ansiedade do Instituto de Psiquiatria do Hospital das Clínicas de São Paulo, registrou que cerca de $25 \%$ da população brasileira sofre de algum transtorno de ansiedade.

A linha entre ansiedade normal e a ansiedade patológica é tênue, e uma pessoa que tenha uma reação ansiosa inadequada e/ou extrema ou de longa duração a um determinado acontecimento pode estar sofrendo de algum tipo de transtorno de ansiedade (D'EL REY, 2005, p. 379).

Sakabe (2002) aponta que, os principais transtornos de ansiedade, segundo o Manual Diagnóstico e Estatístico de Transtornos Mentais DS-MIV (APA, 1994), são classificados em: agorafobia, ataque de pânico, fobia específica, fobia social, transtorno obsessivo-compulsivo, transtorno de estresse pós-traumático, transtorno de estresse agudo, transtorno de ansiedade por condição médica geral, transtorno de ansiedade induzido por substância, transtorno de ansiedade generalizada e transtorno de ansiedade sem outra especificação.

Podem ocorrer casos em que diferentes transtornos estão presentes ao mesmo tempo e não se consegue identificar o que é primário e o que não é, sendo mais certo aludir que esse paciente apresenta mais de um diagnóstico coexistente, uma comorbidade (CASTILLO et al., 2000, p. 20).

Nesse contexto, "O transtorno de ansiedade é responsável por sofrimento individual, familiar e também por grandes custos sociais" (STEIN; SEEDAT, 2004, p. 10). Bystritsky (2006) relata que os transtornos de ansiedade têm um sério impacto na saúde, impacto que não se aplica somente ao gasto com o tratamento, mas também, pelo elevado custo das avaliações médicas e o tratamento de manifestações físicas do distúrbio.

Há que se destacar que "Os dois principais componentes do tratamento dos transtornos de ansiedade são o emprego de medicamentos durante médio e longo prazo e a indicação de psicoterapia" (PITTA, 2010, p. 6). Uma orientação específica ou a associação de ambas depende da avaliação de cada paciente, considerando que essa avaliação diagnóstica deve analisar a gravidade dos sintomas e o grau do prejuízo social.

\section{Fobias}

No estudo das fobias, faz-se necessário uma compreensão do medo tido como não patológico, a fim de elucidar 
as diferenças para com o patológico. $\mathrm{O}$ medo natural, para André (2007a), tem como cargo atrair a atenção do indivíduo para um determinado problema, de tal modo que proporcione acionamento com um motivo razoável, ante um perigo autêntico e não perante mera casualidade de risco, ou de uma memória de um perigo. $\mathrm{O}$ medo dito natural é adequado ao risco, permitindo que o indivíduo reaja da maneira em que está adaptado à situação. Há ocasiões em que o medo exibe-se falso, mas esses alarmes falsos devem se apresentar, ocasionalmente de forma controlável, para que se mantenha um nível normal de medo.

No caso das fobias, o medo manifesta-se sem um perigo iminente ou, até mesmo, sem um agente razoável de estresse. Pode tratar-se de algo imaginário ou somente uma recordação de um acontecimento, caracterizando-se como desproporcional ao risco oferecido. De modo sintetizado, André (2007b) esclarece a diferença entre o medo e a fobia. Segundo ele a fobia é um medo intenso que pode resultar em pânico, tornando-se, muitas vezes, um medo absurdo e irreprimível, o que acaba inibindo as ações do indivíduo que busca o evitamento ou a fuga do encontro com os objetos ou com as situações que invocam esse sentimento. Para Santoro (2008), a fobia não consiste no temor de um objeto ou de uma situação, mas no impedimento de um objeto susceptível de desencadear o temor.

Os quadros fóbicos, segundo Gurfinkel (2001), são analisados pela psiquiatria desde 1870 , porém, ganharam diagnósticos próprios na Classificação Internacional de Doenças (CID) somente em 1947 e na Classificação da Associação Psiquiátrica Americana (DSM), em 1952. Foi com os estudos da Psicanálise que a fobia deixou de ser exclusividade do campo da psicopatologia, deixando de ser também descritiva das manifestações sintomáticas, como afirma Patti e Meneses (2005), abrindo, assim, possibilidades de entendimento mais amplo por meio de sua dinâmica de produção e abordagem terapêutica.

As fobias são específicas quando deliberadas pelo medo excessivo e inflexível pertinente a uma determinada situação ou objeto. De acordo com o CID-10 (1993), são fobias restringidas às circunstâncias altamente particulares, como a proximidade de determinados animais, locais elevados, escuridão, espaços fechados, ingestão de determinados alimentos, ver sangue ou ferimentos, mesmo que a situação desencadeante seja inofensiva, o contato com essa pode desencadear um grau de pânico, como a agorafobia ou fobia social.

As fobias podem ser consideradas "sociais" quando o indivíduo sente um medo persistente e excessivo perante situações em que sinta-se exposto à avaliação de outros, ou que possa correr o risco de comportar-se de modo vergonhoso ou degradante. Descrito no DSM-IV-TR (APA, 2002), o transtorno de ansiedade social (também conhecido como fobia social) é caracterizado como um medo de situações sociais ou de desempenho decorrente do receio de passar vergonha.

As fobias desenvolvem sintomas patológicos reais como o descontrole e os sintomas de ansiedade, os quais podem resultar em taquicardia, sudorese, 
medo paralisante, sensação de desmaio, aversão, pânico e angústia. Para Etkin e Wager (2007), o medo e a prevenção do disparo de sinais ansiosos são corriqueiros a muitos transtornos de ansiedade e, assemelham-se às respostas de excitação, na tentativa de impedir que o sujeito entre em contato com contextos que estejam relacionados aos sinais de medo condicionado.

No caso da gerascofobia, trata-se de um quadro fóbico específico, no qual o indivíduo desenvolve um receio patológico do fator envelhecimento, alheio a precauções. Segundo Poli Neto e Caponi (2007), a Sociedade Internacional de Cirurgia Plástica Estética (ISAPS) e a Sociedade Brasileira de Cirurgia Plástica (SBPC), indicam que o Brasil ocupa o terceiro lugar em quantidade de cirurgias plásticas estéticas no mundo, atrás apenas dos Estados Unidos e do México, pois os métodos de prevenção a sinais de velhice apenas camuflam a idade, contudo, não inibem o seu retardamento.

\section{0 envelhecer}

Camarano (2002) estima, que nos últimos anos, houve um aumento da expectativa de vida brasileira, resultante dos avanços na saúde, na redução da natalidade e na diminuição da mortalidade.

Uma população torna-se mais idosa à medida que aumenta a proporção de indivíduos idosos e diminui a proporção de indivíduos mais jovens, ou seja, para que uma determinada população envelheça, é necessário haver também uma menor taxa de fecundidade (NASRI, 2008, p. 1).

Segundo o censo demográfico divulgado pelo Instituto Brasileiro de Geografia e Estatística (IBGE), até o final da década de 1970, a estrutura etária da população brasileira apresentava o perfil de uma população predominantemente jovem. A tendência é um estreitamento da base da pirâmide, com reduções significativas do número de crianças e jovens no total da população, como é demonstrado na Figura 1.

Figura 1 - Proporção de crianças, jovens-adultos e de idosos em relação ao total da população Brasil - 1940/2050

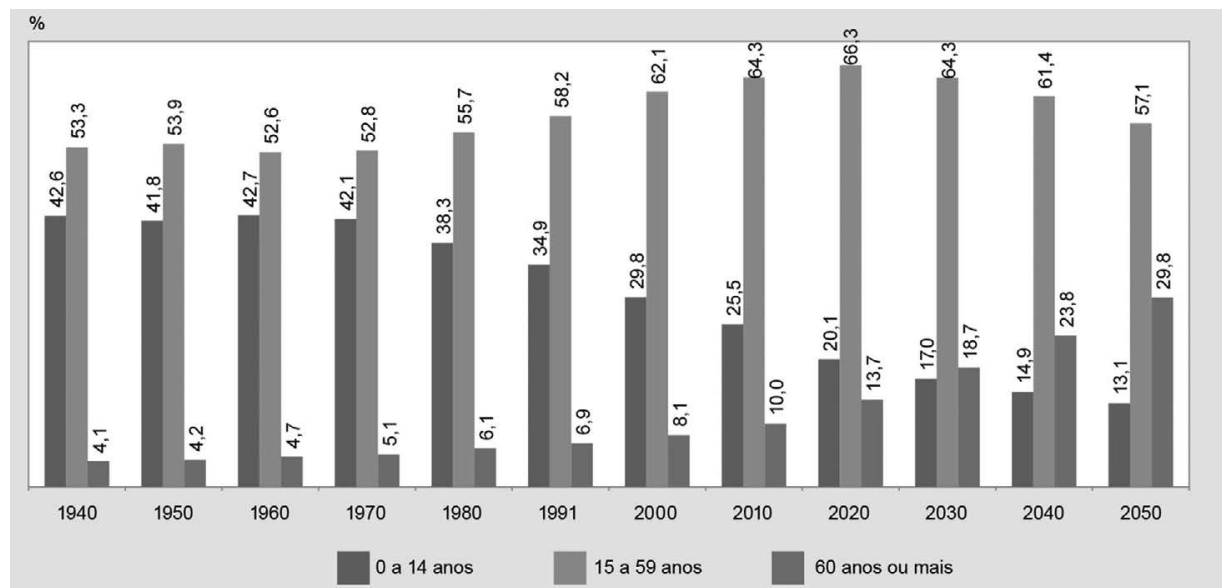

Fonte: IBGE, Censo Demográfico 1940/2000, Revisão 2008. 
Camarano e Pasinato (2004) apontam que nos países em desenvolvimento e, especificamente no caso brasileiro, o acelerado processo de envelhecimento está incidindo em meio a uma conjuntura recessiva e a uma crise fiscal,dificultando a expansão do sistema de proteção social para todos os grupos etários, e em particular, para idosos. O envelhecimento da população brasileira, "é um dos grandes desafios a serem enfrentados, pois se estima que no ano de 2025 o Brasil terá 30 milhões de pessoas com mais de 60 anos (aproximadamente 15\% da população) e será o sexto país em número de idosos" (AREOSA; BULLA, 2008, p. 2).

Nas palavras de Ballone, "O envelhecimento é um fenômeno biológico, psicológico e social que atinge o ser humano na plenitude de sua existência, modifica a sua relação com o tempo, seu relacionamento com o mundo e com sua própria história" (2000, p. 2). Logo, ao envelhecer, qualquer indivíduo sofrerá um declínio das capacidades físicas, psicológicas e comportamentais. Esse processo, segundo Lima (2006), depende de vários fatores, como a adaptação, os relacionamentos familiares e sociais, a posição econômica, o curso de vida e a opinião que se tem de si próprio. Freitas et al. (2010a) apontam que, como todas as situações humanas, a velhice tem uma dimensão existencial, que modifica a relação do sujeito com o tempo, gerando mudanças em suas relações com o mundo e com sua própria história. Assim, a velhice não poderia ser compreendida senão em sua totalidade, mas também como um fato cultural.
Mas o que leva algumas pessoas a aceitarem o processo de envelhecimento com tranquilidade, enquanto outras desenvolvem medos, anseios, preocupações e até mesmo fobia? Para Messina (2003), estaria em jogo o medo de sofrer diante do que é estranho ao corpo envelhecendo (simbolicamente-fisiologicamente), e também, o medo do desconhecido, pertencente a todo neurótico que resiste em romper com a repetição sintomática. $\mathrm{O}$ medo de sofrer pode delinear uma depressão.

Segundo Moreira e Nogueira (2008), estamos vivendo em uma sociedade marcadamente individualista, narcísica, exibicionista e pouco solidária, na qual o envelhecimento é investido de valores negativos, tornando o velho, a velhice e o envelhecer algo indesejável e gerador de sofrimento. Enquanto a juventude é fortemente enaltecida, a velhice é excluída e estigmatizada. Na sociedade capitalista, o velho perde seu poder como produtor de bens e riquezas e como consumidor e, consequentemente, perde seu valor social. Essas são as razões pelas quais a gerascofobia tem amedrontado um número cada vez maior de pessoas.

\section{Gerascofobia, o medo de envelhecer}

O advento da velhice trouxe um problema social nas regiões ocidentais que supervalorizam a jovialidade. Com a propaganda antienvelhecimento das últimas décadas, o desprezo pelos idosos e pela fase senil se intensificou e "a cada dia aumenta-se o interesse pelo envelhecimento bem-sucedido, com ótima qualidade de vida" (BATISTONI; NAMBA, 
2009, p. 734). O aumento da longevidade e do número de aposentados também assumiu uma proporção maior frente aos mais jovens, mas mesmo assim, encontramos indivíduos aposentados que não se designam idosos ou próximos à velhice. De acordo com Batistoni e Namba,

[...] em concordância com o dito popular "você só é tão velho quanto você se sente", um dos achados mais replicados em investigação de idade etária é a de que indivíduos mais velhos tendem a não se sentir velhos. Apesar dessa tendência apontada, a literatura gerontológica tem apontado diversos parâmetros utilizados pelos idosos para derivar seu senso subjetivo de idade, entre eles, parâmetros pessoais, sociais e contextuais (2009, p. 736, grifos do original).

Drummond (2004) aponta que alguns indivíduos buscam compreender o inexorável processo do envelhecimento como um processo gradual, multifatorial e multidiferencial, havendo, atualmente, os que defendem que os aspectos negativos do envelhecimento devem ser enfrentados. Preocupar-se um pouco com idade é comum a todos os seres humanos, porém, de acordo com Almeida e Azevedo (2010) a preocupação torna-se um medo persistente, anormal e injustificado, tem-se uma fobia, denominada de gerascofobia. Essa doença atinge pessoas com boa saúde, do ponto de vista físico e financeiro. É associada a fatores históricos da vida pessoal, como existência de fobias anteriores, falta de realização pessoal e até questões financeiras. Manifesta-se, frequentemente, em jovens e adultos que temem a chegada da velhice e não em sujeitos de idade avançada, o que seria de se esperar.
Muitos fatores são desencadeantes dessa fobia, o medo da morte é um deles. Quem sofre com esse medo, geralmente se angustia com perguntas como: "quando vou morrer? Como vou morrer? Será que vou sofrer muito?" Segundo Delalibera,

A questão da morte está presente em todos os momentos, nas mitologias, no ritual, no inconsciente. Ela é banida das conversas, obscurecida por metáforas e escondida das crianças, a quem é negado o conhecimento da realidade da morte em seus círculos familiares. Os velhos também são afastados por serem seres enrugados, curvados, decrépitos, capazes de transmitir a ideia de decadência e morte (2005, p. 24).

Conforme afirma Delalibera (2005), cada sociedade confere à morte o seu significado próprio, mas todas respondem ao problema da morte referindo-se ao símbolo que o corpo é. $\mathrm{O}$ que se teme na morte é, exatamente, o que ela tem de morte, por isso busca-se dar ao cadáver a aparência de vida, vestindo-o, maquiando-o, dando-lhe uma boa aparência.

Nesse contexto, o corpo, então seria outro motivo de preocupação e um fator importante que caracteriza a gerascofobia. O medo do envelhecimento surge do medo de marginalização ou de rejeição e, segundo Souza et al. (2010), embora as sociedades, de um modo geral, atribuam significados distintos às etapas da vida, com funções e importâncias diferenciadas, é na sociedade capitalista que a velhice é percebida como a pior fase da vida. O idoso nessa sociedade é o velho, não o ancião.

Essa visão de decadência e finitude é uma forte influência da mídia, e da concepção que as pessoas, principalmente do 
Ocidente, têm dos idosos. Enquanto no Oriente, o idoso é valorizado e respeitado como símbolo de experiência e sabedoria, no Ocidente, o envelhecimento é sinônimo de perda das capacidades e da beleza. Em relação ao corpo, explica Castro,

A segunda década do século $X X$ foi crucial na formulação de um novo ideal físico, tendo a imagem cinematográfica interferido significativamente nessa construção. No fim da década, mulheres, sob o impacto combinado das indústrias do cosmético, da moda, da publicidade e de Hollywood, incorporam o uso da maquiagem, principalmente o batom, e passam a valorizar o corpo esbelto, esguio (2001, p. 2).

A população é, constantemente, bombardeada com informações, propagandas e anúncios alusivos, cuja ideia central é que para ser bem aceito na sociedade, $o$ sujeito precisa ser jovem e bonito. Isso faz do tempo um grande rival das pessoas, acentuando o medo de envelhecer. Como afirma Guerra e Caldas (2010), o idoso, no contexto da mídia, é apresentado como "problema" e apontado sob o olhar do outro, desconsiderando a sua opinião quanto ao momento que vive dentro do processo de envelhecimento e suas vivências e evidências a respeito de si mesmo, demonstrando o descarte da sua autopercepção como indivíduo nesse processo.

O processo de amadurecimento psicológico também influi diretamente no aparecimento da gerascofobia. Freitas et al. (2010b) destacam as perdas na velhice, tais sejam, o surgimento de doenças, a morte de amigos e familiares, a viuvez e a ausência de papéis sociais valorizados, como a preocupação com o trabalho e a condição financeira. Essas perdas tem a capacidade de afetar a autoestima do idoso, determinando o surgimento de situações de crise e até mesmo de depressão.

Os gerascofóbicos afetados, geralmente, são pessoas que permanecem ao longo do curso da vida sem atingir seus objetivos pré-fixados e não correspondem a suas expectativas idealizadas. Para Almeida e Azevedo (2004), o perfil do afetado pela gerascofobia tem como características traços de personalidade ansiosa, histérica ou narcisista. Além disseo, os indivíduos que sofrem dessa fobia supervalorizam bens materiais, tem dificuldade para lidar com a perda do poder de sedução ou de riqueza.

A gerascofobia apresenta-se com manifestações de ansiedade, medo e constantes pensamentos negativos, os quais levam o indivíduo a demonstrar um verdadeiro medo quando se pensa em velhice.

Apresentam possíveis casos de gerontofobia, que é o medo de pessoas idosas, e em muitas ocasiões desprezo por pessoas da faixa etária senil (PAIVA, 2008, p. 56).

Rifiotis (2007, p. 147) cita Erikson (1990) ao mencionar a importância do ciclo de vida, fala do processo de envelhecer como uma percepção do tempo, manifestando um sentido, não apenas para a terceira idade, mas para todo o ciclo vital, o tempo em todos os estágios da vida. Afirma também que, é preciso se preparar para uma nova condição do idoso, para uma reestruturação da vida, de dar novos significados para a relação de um começo e de um fim de vida.

Entre as possíveis maneiras de retardar a velhice, segundo Lima (2006) 
destaca-se viver criativamente, adquirindo novos conhecimentos, criando novos hábitos adaptados ao momento presente; aceitar a senectude como uma fase natural da vida; não se deixar dominar pelo medo, pelo impulso à inatividade, à autopiedade, à ausência de afetividade, à falta de interesse e coragem; reconhecer que a saúde física e psíquica é consequência da capacidade de agir de acordo com seu verdadeiro interesse; compreender que somos nosso próprio artesão - damos forma à nossa existência.

De acordo com Blazer (2006), a ideia de que a velhice pode ser uma fase agradável da vida não é algo novo, e há milênios foi executado, paralelamente, a uma visão mais negativa do envelhecimento. $\mathrm{O}$ modelo mais popular de envelhecimento bem-sucedido foi desenvolvido por Rowe e Kahn (1997), que o definem como elevado grau de função física e cognitiva, engajamento ativo e a ausência de doença. Segundo Silvia e Santos (2010), as experiências de manter o bem-estar e/ou de lidar com o adoecimento são constantes na vida dos indivíduos que enfrentam o envelhecimento, visto ser necessário promover a saúde e estimular comportamentos, visando à manutenção da autonomia e ao envelhecimento bem-sucedido. Para Meyer (2009), é de grande importância compreender melhor a população crescente de adultos, e assim, identificar as características das pessoas que mantêm elevada qualidade de vida e bem-estar em relação à longevidade de suas vidas, podendo levar a intervenções eficazes e mudanças da vida real para os outros.

\section{Considerações finais}

Ao depararmos-nos com um aumento significativo no número de idosos e a radical mudança na pirâmide social, que cada vez mais se assemelha às dos países europeus, voltamos nossos olhos a um assunto que não é tão comentado ou explicado cientificamente como deveria ser, mas é de extrema importância para o entendimento e saber humano, o envelhecimento.

Sendo assim a velhice um processo comum a todos, atingindo homens e mulheres de uma forma inevitável, buscamos compreender quais as preocupações e danos que essa causa na população que está envelhecendo, visto que vivemos em uma sociedade que privilegia o jovem e cultua o corpo bonito, "sarado", magro e em forma.

Os indivíduos experimentam uma crescente preocupação com a imagem e a estética, a qual está relacionada à ideologia narcisista social que vivemos atualmente, graças à influência da mídia. Quando essa preocupação com a imagem, com o corpo, com o ser jovem, bonito e fisicamente atraente é exagerada, pode tornar-se uma patologia.

Quando esse medo afeta a vida de um indivíduo, causando tensões, angústias e preocupações exageradas, levando-o a buscar, de qualquer maneira, a "fonte da juventude", sem considerar os riscos que corre, configura-se em um transtorno de ansiedade, uma fobia mais especificamente, chamada de gerascofobia, o medo de envelhecer. Como qualquer fobia, a gerascofobia acarreta um intenso sofrimento, medo e constantes pensamentos 
negativos que levam o indivíduo a demonstrar um medo verdadeiro quando pensa em velhice.

Para que o amadurecimento ocorra de maneira saudável, precisamos organizarmo-nos. Antecipadamente, para o advento da melhor idade, permitindo que essa nova fase do ciclo da vida chegue, apropriadamente, de maneira confortável e segura. Esta preparação auxilia não apenas aos que já são idosos, mas também ampara os que estão em processo de envelhecimento.

\section{Gerascophobia - the fear of aging in contemporary}

\section{Abstract}

This study aims to contribute to psychological science about the advent of old age, the implications related to a narcissist social ideology which we now live mediainduced, also stressing the importance of this issue for other fields of study, such as gerontology and geriatrics. The survey, according to a literature review, about the rise in the elderly population in the Brazilian socio-demographic pyramid and the experience the aging process, where the concept that is lived in a stigmatized way, comes to represent a threat to self-acceptance, making people become vulnerable to mental suffering of all kinds and even pathologies. The fear of aging is not only associated with senile phase, to a gerasphobic the awakening of the disease is a relevant fear of what awaits them at maturity, creates a pure fear over the course of the life cycle, not understanding the natural consequences that's addresses the process of aging, risking several ways to slow the signs of aging. It is realized, then, the presence of actual physiological symptoms of pathological anxiety, characterized as an exaggerated fear that can affect an individual's life, causing tensions, anxieties and exaggerated concerns, leading to seek at any cost "fountain of youth" without putting into account the risks related to it.

Keywords: Gerascophobia. Phobia. Grow old. Anxiety disorder.

\section{Referências}

AMERICAN PSYCHIATRIC ASSOCIATION. Manual de diagnóstico e estatística dos distúrbios mentais (DSM-IV). Porto Alegre: Artes Médicas, 1994. 938p.

ANDRÉ, C. Psicologia do medo: como lidar com temores, fobias, angústias e pânicos. Tradução de João Batista Kreuch. Petrópolis: Vozes, 2007. 304p.

ALMEIDA, G; AZEVEDO, M. Medo de envelhecer. Revista Eclética, Rio de Janeiro, v. 1 , n. 30 , p. $12-16$, jun. 2010.

AREOSA, S. V. C; BULLA, L. C. Contexto social e relações familiares: o idoso provedor. 2008. Tese (Doutorado em Serviço Social) - Pontifícia Universidade Católica do Rio Grande do Sul, Porto Alegre, 2008.

BALLONE, G. J. Transtornos do envelhecimento. In: Psiqueweb. Programa de Psiquiatria Clínica na Internet. Campinas, 2000. Disponível em: <http://www.psiqweb.med. br>. Acesso em: 13 maio 2012.

BATISTONI, S. S; NAMBA, C. S. Idade subjetiva e suas relações com o envelhecimento bem-sucedido. Psicologia em Estudo, Maringá, v. 15, n. 4, p. 733-742, 2010.

BLAZER, D. G. Successful aging. American Journal of Geriatric Psychiatry. Philadelphia, v. 14, n. 1, p. 2-5, jan. 2006.

BORGES, C. N; BUENO, M. G.; LIMA, T. M. Consumo, estética e saúde feminina nas páginas e discursos da revista Boa Forma. In: CONGRESSO DE CIÊNCIAS DA COMU- 
NICAÇÃO, 15, 2012, Campo Grande. Anais... Campo Grande: Interfaces, 2012, p. 1-12.

BOURNE, E. J. Acabe com a ansiedade antes que ela acabe com você. São Paulo: Gente, 2008. 192p.

BYSTRITSKY, A. Treatmant-resistant anxiety disorders. Molecular Psychiatric, Los Angeles, v. 11, p. 805-814, jul. 2006.

CAMARANO, A. A. Envelhecimento da população brasileira: uma contribuição demográfica. In: FREITAS, E. V.; PY, L.; NERI, A. L.; CANÇADO, F. A. X.; GORZONI, M. L.; ROCHA, S. M. (Eds). Tratado de Geriatria e Gerontologia, Rio de Janeiro: Guanabara-Koogan, 2002. p. 58-71.

CAMARANO, A. A; PASINATO, M. T. O envelhecimento populacional na agenda das políticas públicas. In: CAMARANO, A. A. (Org). Os novos idosos brasileiros muito além dos 60? Rio de Janeiro: IPEA, 2004.

CASTILLO, A. R; RECONDO, R; ASBAHR, F.R; MANFRO, G. G. Transtornos de ansiedade. Revista Brasileira de Psiquiatria, São Paulo, v. 22, n. 2, p. 20-23, dez. 2000.

CASTRO, A. L. O culto ao corpo: identidades e estilo de vida. Annablume, São Paulo, v. 22, n. 2, p. 20-23, 2000.

DALGALARRONDO, P. Psicopatologia e semiologia dos transtornos mentais. 2. ed. Porto Alegre: Artes Médicas, 2000. 438p.

DELALIBERA, A. M. A imagem do corpo $e$ a angústia sobre o corpo no envelhecer e no morrer. 2005. Trabalho de conclusão de curso (Graduação em Psicologia) - Universidade Federal de São Carlos - UFSCAR, São Carlos, p. 7-49, nov. 2005.

D`EL REY, G. J. F. Quando a ansiedade torna-se uma doença? Integração, São Paulo, v. 6 , n. 43 , p. 379-382, 2005.

DRUMMOND, A. J. E. Procurando superar a modelização de um modo de envelhecer. Movimento, Porto Alegre , v. 10, n. 2, p. 57-71, maio/ago. 2004.
ETKIN, A.; WAGER, D. Functional neuroimaging of anxiety: a meta-analysis of emotional processing in PTSD, social anxiety disorder, and specific phobia. American Journal Psychiatry, Bethesda, v. 1, n. 164, p. 1476-1488, out. 2008.

FREITAS, M. C.; QUEIROZ, T.; SOUZA, J. A. O significado da velhice e da experiência de envelhecer para os idosos. Revista Escola Enfermagem, São Paulo , v. 44, n. 2, p. 407412, 2010.

GUERRA, A. C. L; CALDAS, C. P. Dificuldades e recompensas no processo de envelhecimento: a percepção do sujeito idoso. Ciência e saúde coletiva, Rio de Janeiro , v. 15 , n. 6, p. 2931-2940, 2010.

GURFINKEL, A. C. Fobia clínica psicanalítica. São Paulo: Casa do Psicólogo, 2001. 167p.

IBGE. Coordenação de populacão e indicadores sociais. Perfil dos Municípios Brasileiros: gestão pública. 2008. Rio de Janeiro: IBGE, 2008. 219p.

LIMA, L. H. P. Eu e o envelhecimento. Porto Alegre: AGE, 2006. 96p.

MESSINA, M. Dimensões do envelhecer na contemporaneidade: estados gerais de psicanálise. In: ENCONTRO TEMÁTICO EM GERONTOLOGIA, 2,2002, Rio de Janeiro, 2002.

MEYER, S. V. Successful aging: a quantitative study of resiliency and adaptability as mediating factors in the successful aging of older adults. 2009. These (Doctor in Phisicology). Oregon, - Pacific University, 2009.

MOREIRA, V; NOGUEIRA, F. Do indesejável ao inevitável: a experiência vivida do estigma de envelhecer na contemporaneidade. Psicologia USP, São Paulo, v. 19, n. 1, p. 59-79, jan./mar. 2008.

NARDI, A. E.; FONTENELLE, L. F.; CRIPPA, J. A. S. New trends in anxiety disorders. Revista Brasileira de Psiquiatria, São Paulo , v. 34, p. 505-508, 2012. 
NASRI. F. O envelhecimento populacional no Brasil. Eistein, São Paulo, v. 6, n. 1, p. 4-6, 2008.

ORGANIZAÇÃO MUNDIAL DA SAÚDE OMS. Classificação Estatística Internacional de Doenças e Problemas Relacionados à Saúde-CID-10. 2008. Disponível em: <http// www.datasus.gov.br/cid10/v2008/cid10. html>. Acesso em: 13 ago. 2012.

PAIVA, J. L. Qualidade de vida dos seniores: estudo comparativo entre as três freguesias do concelho de Portimão. 2008. Dissertação (Mestrado em Serviço Social) - Universidade de Coimbra, Coimbra - Portugal, 2008.

PATTI, E. A. M. R; MENESES, I. C. Crianças com sintomas fóbicos e o tratamento odontológico. Revista Científica da Universidade de Franca, São Paulo, v. 5, n. 1, p. 1-9, dez. 2005.

PITTA, J. C. N. Como diagnosticar e tratar transtornos de ansiedade. Anxiety disorders, São Paulo: Moreira Jr , v. 68, n. 12, p. 6-13, dez. 2010.

POLI NETO, P.; CAPONI, S. N. C. The 'medicalization' of beauty. Interface Comunicação, Florianópolis , v. 11, n. 23, p. 579-585, dez. 2007.

RIFIOTIS, T. O idoso e a sociedade moderna: desafios da gerontologia. Pró-posições, Campinas (SP), v. 18, n. 1, p. 137-151, jan./ abr. 2007.

ROWE, K. W.; KAHN, R. L. Sucessful aging. The Journals of Gerontology, New York, v. 37, n. 4, p. 433-440, 1997.

SAKABE, G. O transtorno do pânico: as consequências do diagnóstico tardio. 2002. Trabalho de conclusão de curso (Graduação em Medicina) - Universidade do Oeste Paulista - Unoeste, Presidente Prudente: 2002. um caso de fobia. Reverso, Belo Horizonte, v. 30 , n. 56 , p. $77-83$, out. 2008 .
SANTORO, V. C. A dama da lagartixa:

SILVA, A. C. S.; SANTOS, I. Promoção do autocuidado de idosos para o envelhecer saudável: aplicação da teoria de Nola Pender. Contexto Enfermaria, Rio de Janeiro , v. 9, n. 4, p. 745-753, set. 2010.

SILVA, C. R. Ansiedade no meio escolar. 2011. Trabalho de conclusão de curso ( Graduação em Biologia) - Universidade de Brasília, Brasília, 2011.

SOUZA, R. F.; MATIAS, H. A.; BRETAS, A. C. Reflexões sobre envelhecimento e trabalho. Ciência e saúde coletiva, São Paulo, v. 15, n. 6 , p. $2835-2843,2010$.

SOUZA, R. F.; SKUBS, T.; BRÊTAS, A. C. P. Envelhecimento e família: uma nova perspectiva para o cuidado de enfermagem. Revista Brasileira de Enfermagem, Brasília, v. 60 , n. 3, p. 263-267, jan. 2007.

STEIN, D. J.; SEEDAT, S. Unresolved questions about treatment-resistant anxiety disorders. CNS Spectrums, Cape Town (South Africa), v. 9, n. 10, p. 715, 2004.

VERAS, R. Envelhecimento populacional contemporâneo: demandas, desafios e inovações. Revista Saúde Pública, Rio de Janeiro, v. 43, n. 3, p. 548-554, abr. 2009.

WITTCHEN, H. U; FEHM L. Epidemiology, patterns of comorbidity, and associated disabilities of social phobia. Psychiatric Clinics of North America, Dresden (Germany), v. 24, n. 4, p. 617-641, dez. 2001

ZAMIGNANI, D. R; BANACO, R. A. Um panorama analítico-comportamental sobre os transtornos de ansiedade. Revista Brasileira Terapia Comportamental Cognitiva, São Paulo, v. 7, n. 1, p. 77-92, 2005. 